\title{
Pulsatile luteinizing hormone disruption in depression
}

\author{
Patricia Grambsch ${ }^{a, *}$, Elizabeth A. Young ${ }^{b}$, William H. Meller
}

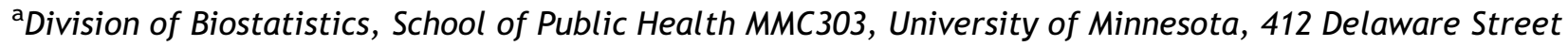
SE, Mayo A-460, Minneapolis, MN 55455, USA

${ }^{\mathrm{b}}$ Department of Psychiatry and Mental Health Research Institute, University of Michigan Ann Arbor, MI, USA

'Department of Psychiatry, University of Minnesota Minneapolis, MN 55455, USA

Received 4 February 2003; accepted 16 July 2003

\section{KEYWORDS}

Hypothalamic-pituitaryovarian axis; Spectral analysis; Periodogram

\begin{abstract}
Summary Two independent groups recently published data comparing pulsatile luteinizing hormone (LH) release between depressed and control women. Despite similar populations and LH sampling frequency, they reached different conclusions: Meller et al. [Am. J. Psych. 154 (1997) 1454] found disruption of normal LH pulsatility in depressed women, whereas Young et al. [Arch. Gen. Psych. 57 (2000) 1157] did not. To resolve this discrepancy, the current study applies a single, well-established statistical method, spectral analysis, to the two data sets and concludes that both depressed populations display significantly altered LH pulsatile release.
\end{abstract}

(C) 2003 Elsevier Ltd. All rights reserved.

\section{Introduction}

Hypothalamic control of a variety of endocrine functions is impacted by major depressive disorder. Despite its potential clinical relevance, the least investigated is the hypothalamic-pituitaryovarian (HPO) axis. Releasing factors, trophic and steroid hormones and end organ receptors must be synchronized for normal ovulatory function. Luteinizing hormone (LH), a sensitive marker of HPO function, consistently exhibits a subcirchoral rhythm of pulse release (roughly every $65-85 \mathrm{~min}$ ) in the follicular phase of normal menstruating women during daylight hours. This rhythm is not

"Corresponding author. Tel.: +1-612-624-0418; fax: +1-612626-0660.

E-mail address: pat@biostat.umn.edu (P. Grambsch). maintained in the luteal phase, when pulses are quite sparse and irregular (Johnson and Everitt, 2000).

Recently, two independent research groups have examined the impact of depression on the pulsatile release of $\mathrm{LH}$ in premenopausal women. Despite similar populations and LH sampling frequency, their studies reached different conclusions. Young et al. (2000) found no differences in LH pulsatility between normal control and depressed women, whereas Meller et al. (1997, 2001) found disruption of the normal subcirchoral rhythm in the depressed group. The purpose of this paper is to see if these discrepancies can be resolved by the application of a single statistical method, spectral analysis, to the two data sets. 


\section{Methods}

\subsection{Comparison of Young and Meller study designs and populations}

Both studies recruited women of childbearing age. Depressed vs. control status was determined by a standard structured clinical interview (SCID for Young and SADS for Meller). The inclusion and exclusion criteria were similar. Both studies excluded anyone on psychotropic medication or oral contraception and anyone with a current or recent history of alcohol or drug abuse. The depressed women could not be taking any antidepressant medication and could have no other axis I disorders.

The study designs differed in several respects. The Young study recruited women at all stages of the menstrual cycle. Depressed and control women were paired on the basis of age and day of period. The Meller study recruited only women in the early- to mid-follicular stage, two to seven days after commencement of menstrual bleeding. There was no additional pairing. Day of period was determined from participant report in both studies. In addition, the Young study used serial q30 min estradiol and progesterone to confirm the menstrual cycle phase, luteal or follicular. The Meller data set had 50 participants: 24 controls and 26 depressed. The Young data had 12 control depressed pairs in the follicular phase and 13 pairs in the luteal phase. Both studies sampled serum LH every $10 \mathrm{~min}$. The Young study had a longer sampling period, $12 \mathrm{~h}$ from 09:00 to 20:50 h, obtaining 72 data points per participant. The Meller study sampled from 11:00 to 19:00 h, with 49 sample points per participant. Finally, the Young study assayed LH by chemiluminescence, whereas the Meller study used an enzymatic-immune assay.

\subsection{Spectral analysis}

The spectral analysis of a time series, such as the LH concentrations, measured at equally spaced intervals, partitions the series variability into harmonic components. These are sine waves, each with one of the Fourier frequencies. The Meller data (49 points) had 24 Fourier frequencies and the Young data (72 points) had 36 Fourier frequencies. A sinusoid with the $k$ th Fourier frequency completes precisely $k$ cycles in the time span of the data. So, in the Young data, these frequencies included $1 / 12$ cycle per hour, 2/12 cycles per hour, etc. in equal increments up to three cycles per hour and in the Meller data, they were roughly $1 / 8$ cycle per hour, $2 / 8$ cycle per hour, up to three cycles per hour. Each Fourier frequency has an amplitude associated with it. Because the sum of the squared amplitudes equals that of the squared deviations from the data series' mean and so is proportional to the variance, each squared amplitude represents the amount of variation in the series attributable to a sinusoidal oscillation at the corresponding Fourier frequency. Spectral analysis results in a periodogram, a graph of the squared amplitudes against the Fourier frequencies, revealing which harmonic components predominate in the data. More detailed information on spectral analysis, both in general and for the LH data, can be found in Diggle's excellent monograph (Diggle, 1990).

Following the recommendations of Murdoch et al. (1985, 1989), who pioneered spectral analysis for LH data, we computed the periodogram of each time series by the fast Fourier transform applied to the residuals from a regression of each $\mathrm{LH}$ time series on a quadratic polynomial on time, to remove any long term trend, and tapered by a split bell cosine taper $(10 \%)$, to ameliorate the leakage problem (Bloomfield, 2000). Each periodogram was scaled so that the sum of the components equaled $100 \%$. Thus, each component represented the percentage of variance explained by that Fourier frequency.

The Young data were treated as two data sets: the luteal phase women were analyzed separately from the follicular phase women. For plotting, the geometric mean of the periodograms for each diagnostic group in each data set was computed and rescaled to sum to $100 \%$. For testing differences in periodograms between diagnostic groups, we used rhythmicity indices as defined by Warner (1998), the percentage of variance accounted for by a set of frequencies of interest, specified by the researcher. These were compared by $t$ tests on the log scale. Paired $t$ tests were used for the Young data, because of the paired design, and two group $t$ tests for the Meller data.

\section{Results}

\subsection{Follicular phase data}

Fig. 1 presents the geometric means of the periodograms. The top panel shows the depressed and control means for the Young data; the bottom panel shows the means in the Meller data. Despite the fact that the Young data set has roughly half as many subjects as the Meller data set and more Fourier frequencies (36 vs. 24), the two panels are similar. In both control mean periodograms, the most noticeable feature is a prominent peak in 


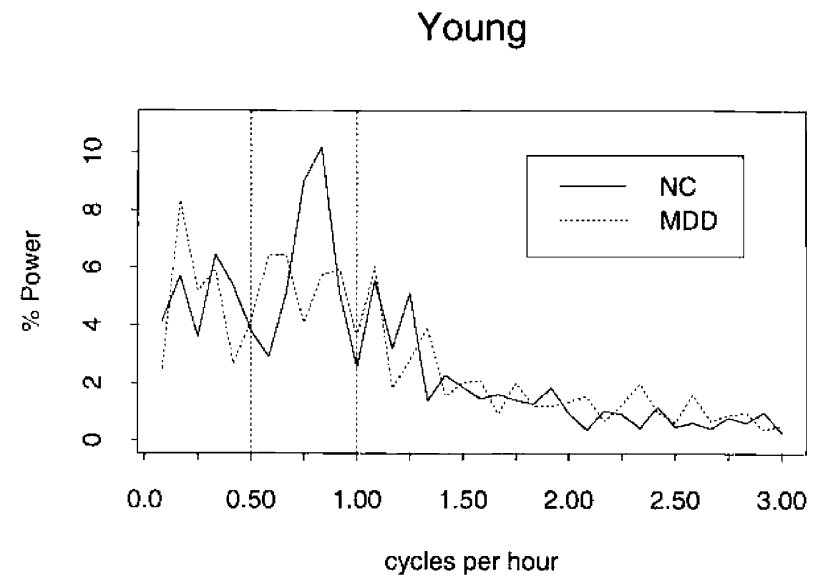

Meller

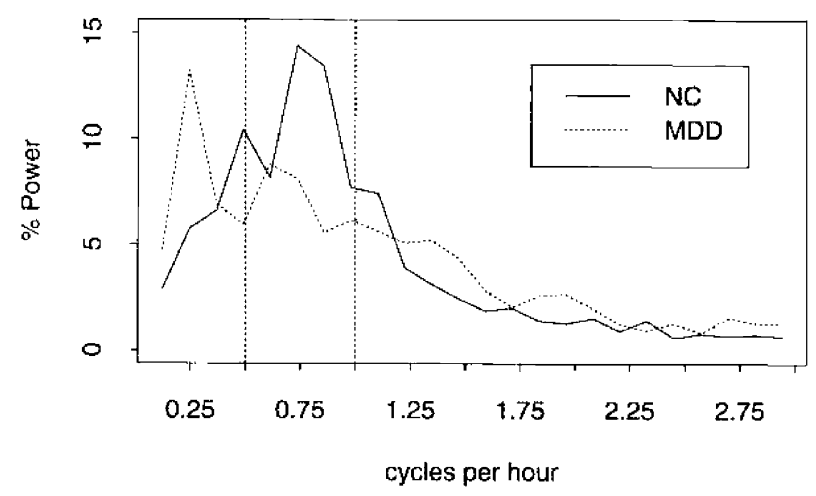

Fig. 1. Geometric mean periodograms of the Young (upper panel) and Meller (lower panel) follicular phase depressed and control groups, scaled to sum to $100 \%$ for each group, based on residuals from a quadratic polynomial on time with $10 \%$ cosine bell taper. The lower is reprinted from Meller et al. (2001), with permission from Elsevier Science.

the subcirchoral region. In the Young data, it covers the Fourier frequencies corresponding to 0.75 and 0.833 cycles per hour, i.e. periods of 80 and $72 \mathrm{~min}$. In the Meller data, the peak involves 0.73 and 0.86 cycles per hour, i.e. 81.67 and $70 \mathrm{~min}$ periods, essentially at the same place. In contrast, the depressed mean periodograms have no prominent peaks in the subcirchoral region in either data set. In the Young data, the rhythmicity index of the sum of the 0.75 and 0.833 cycles per hour harmonic components accounts for $15.0 \%$ of the variance in the controls on average, but $8.8 \%$ for the depressed (log scale paired $t=2.40$, $\mathrm{df}=11, p=0.035$ ). In the Meller data, the rhythmicity index of the sum of the 0.75 and 0.833 cycles per hour harmonic components accounts for $25.9 \%$ of the variance of the controls on average, but $13.6 \%$ for the depressed (log scale $t$ test $=2.75, \mathrm{df}=48, p=0.008$ ). So in both data sets, the rhythmicity index accounts for nearly

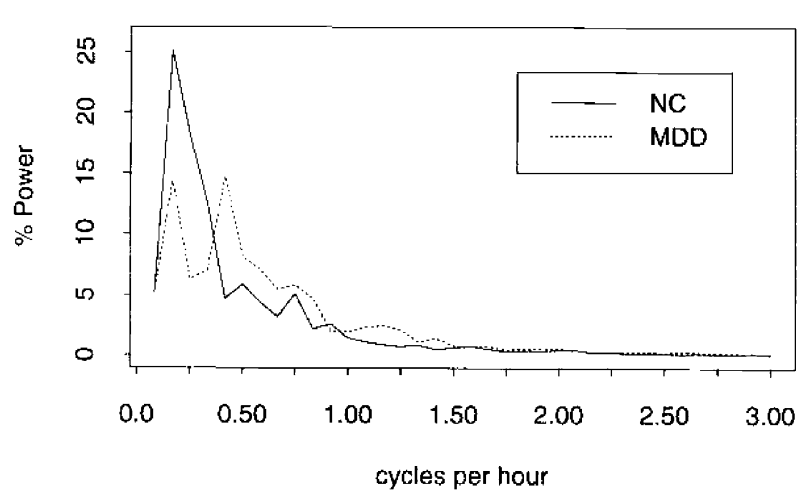

Fig. 2. Geometric mean periodograms of the Young luteal phase depressed and control groups, scaled to sum to $100 \%$ for each group, based on residuals from a quadratic polynomial on time with $10 \%$ cosine bell taper.

twice as much of the variance for the controls as for the depressed.

\subsection{Luteal phase data}

Fig. 2 shows the geometric mean periodograms for the controls and depressed from Young's data. The controls have a single prominent peak at the second Fourier frequency, 0.167 cycles per hour (a $6 \mathrm{~h}$ period). The depressed have two lesser peaks of comparable size, one also at the second Fourier frequency and the other at the fifth, corresponding to 0.417 cycles per hour. Treating each of these frequencies as a rhythmicity index, we find that the second Fourier frequency accounts for $23.6 \%$ of the variance of the controls on average, but $12.6 \%$ for the depressed (log scale paired $t$ test $=2.17, \mathrm{df}=12, p=0.051$ ) and the fifth Fourier frequency accounts for $4.9 \%$ of the control variance, but $13.8 \%$ for the depressed (log scale paired $t$ test $=2.67, \mathrm{df}=12, p=0.021$ ).

\section{Discussion}

Comparing data sets from independent researchers is important. This was possible for the Young and Meller follicular phase data. The well-documented subcirchoral rhythm was found in both normal controls, but was disrupted in both depressed samples. Statistically significant differences between depressed and controls were found in the subcirhoral rhythmicity indices in both data sets and visual examination of the periodograms suggests that the magnitude of the difference was quite similar. The luteal phase was available only in the Young data set. The small size of the data set and the post hoc nature of the rhythmicity 
indices suggest caution. However, it is interesting to note that again we found statistically significant differences between the depressed and controls.

We can contrast the current findings with the original analyses of these data. The Young data were initially analyzed (Young et al., 2000) by a time series pulse detection algorithm, "pulsefit" (Kushler and Brown, 1991), which looks for significant pulses in a background of noise and then characterizes them by summary statistics, including pulse frequency and mean amplitude. These analyses showed no significant differences between depressed and controls in any LH pulsatility measure except for LH half-life, which was shorter in the depressed participants. The Meller data were also analyzed by a pulse detection algorithm, "cluster" (Veldhuis and Johnson, 1986). Statistically significant differences were found in pulse frequency and the standard deviation of pulse amplitude, but only for one of three parameter settings (Grambsch et al., 2002). In contrast to pulse detection algorithms which work in the time domain, spectral analyses are frequency domain analyses and appear to be more sensitive in detecting abnormal rhythms in LH pulsatility, as found in this analysis. Furthermore, the source of these rhythms is brain systems; so frequency domain methods may better reflect abnormalities

in the brain systems controlling the HPO axis in depression.

This comparative reanalysis suggests that major depressive disorder disrupts LH pulsatility in the follicular phase of the menstrual cycle and provides evidence that it may do so in the luteal phase as well. Abnormal control of $\mathrm{LH}$ pulsatile release has important implications at all levels of the HPO axis. LH pulsatility is highly correlated with hypothalamic release of GNRH (Clark and Cummins, 1982; Moenter et al., 1993), which must be maintained within narrow limits for normal HPO functioning (Filicori et al., 1993). Remarkably, pituitary tropic cells respond to high frequency GNRH pulsatility with increased synthesis of the beta subunit of $\mathrm{LH}$, while slower frequency GNRH favors the synthesis of beta FSH (Schwartz, 2000). LH itself must be closely regulated for normal ovarian function. Quintal-Franco et al. (1999) have shown that theca, granulosa, and luteal cells require optimally regulated LH pulsatility for normal corpus luteum structure and function; Duffy et al. (2000) have discovered that LH frequency influences the expression of estrogen receptors. Because LH and FSH pulsatility are both regulated by GNRH and are highly correlated, these data suggest possible FSH abnormalities which could impair folliculogenesis (Kooistra and Okkens, 2001). Therefore, disruption of LH pulsatility has the potential for disturbing a wide variety of HPO functions.

In fact, the original Young data analysis (Young et al., 2000) found significantly decreased levels of estradiol in the follicular phase of the depressed; this decrease may have resulted from the disruption of the follicular subcirchoral rhythm.

\section{Acknowledgements}

Elizabeth A. Young and William H. Meller were supported by the National Institutes of Mental Health, National Center for Research Resources Clinical Research Center under grant numbers $\mathrm{MN}$ 50030, MH-01931, RR-00042 and MH-19815, MH52287, RR-00400, respectively.

\section{References}

Bloomfield, W.A., 2000. Fourier Analysis of Time Series: An Introduction, second ed. Wiley, New York.

Clark, I.J., Cummins, J.T., 1982. The temporal relationship between gonadotropin releasing hormone (GNRH) and luteinizing hormone (LH) secretion in ovariectomized ewes. Endocrinology 111, 1737-1739.

Diggle, P., 1990. Time Series: A Biostatical Introduction. Oxford University Press, Oxford.

Duffy, M., Chaffin, C.L., Stouffer, R.L., 2000. Expression of estrogen receptor alpha and beta in the rhesus monkey during the menstrual cycle's regulation by luteinizing hormone and progesterone. Endocrinology 141, 1711-1717.

Filicori, M., Cognigni, G., Della, P., Arone, R., Sambaturo, M., Falbo, A., Pecorari, R., Carbone, F., Meriggrola, M.C., 1993. Role of gonadotropin releasing hormone secretory dynamics in the control of the human menstrual cycle. Hum. Reprod. 9, 62-65.

Grambsch, P.L., Meller, W.H., Grambsch, P.V., 2002. Periodograms and pulse detection methods for pulsatile hormone data. Statist. Med. 21, 2331-2344.

Johnson, M.H., Everitt, B.J., 2000. Essential Reproduction, fifth ed. Blackwell Science, Oxford.

Kooistra, H.S., Okkens, A.C., 2001. Role of changes in the pulsatile secretion pattern of FSH in initiation of ovarian folliculogenesis in bitches. J. Reprod. Fertil. Suppl. 57, 11-14.

Kushler, R.H., Brown, M.B., 1991. A model for the identification of hormone pulses. Statist. Med. 10, 329-340.

Meller, W., Zander, K., Crosby, R., Tagatz, G., 1997. Luteinizing hormone pulse characteristics in depressed women. Am. J. Psych. 154, 1454-1455.

Meller, W., Grambsch, P.L., Tagatz, G., Bingham, C., 2001. Hypothalamic pituitary gonadal axis dysregulation in depressed women. Psychoneuroendocrinology 26, 253-259.

Murdoch, M.L., Diggle, P.J., Dunlop, W., Kendall-Taylor, P., 1985. Determination of the frequency of pulsatile luteinizing hormone secretion by time series analysis. Clin. Endocrinol. 22, 341-346. 
Murdoch, M.L., Diggle, P.J., White, M.C., Harris, M., KendallTaylor, P., Dunlop, W., 1989. Luteinizing hormone in women is secreted in superimposed pulse patterns. Clin. Sci. 76, 125-135.

Quintal-Franco, J.A., Kojima, F.N., Melvin, E.J., Lindsey, B.R., Zanella, E., Fike, K.E., Wehram, M.E., Clopton, D., Kinder, J.E., 1999. Corpus luteum development and function in cattle with episodic release of luteinizing hormone pulses inhibited in the follicular and early luteal phases of the estrous cycle. Biol. Reprod. 61, 921-926.

Schwartz, N.B., 2000. Neuroendocrine regulation of reproductive cyclicity. In: Conn, P.M., Freeman, M.E. (Eds.),
Neuroendocrinology in Physiology and Medicine. Humana Press, Totowa, NJ.

Veldhuis, J.D., Johnson, M.L., 1986. Cluster analysis: a simple, versatile and robust algorithm for endocrine pulse detection. Am. J. Physiol. 250, E486-E493.

Warner, R.M., 1998. Spectral Analysis of Time Series Data. Guilford Press, New York.

Young, E.A., Midgley, A.R., Carlson, N.E., Brown, M.B., 2000. Alteration in the hypothalamic-pituitary-ovarian axis in depressed women. Arch. Gen. Psych. 57, 11571162. 